\title{
CENTRAL CROSS-SECTIONS MAKE SURFACES OF REVOLUTION QUADRIC
}

\author{
BRUCE SOLOMON
}

\begin{abstract}
When all planes transverse and nearly perpendicular to the axis of a surface of revolution $S$ intersect it in loops having central symmetry, $S$ must be quadric.
\end{abstract}

\section{Dedicated to Sue Swartz}

\section{INTRODUCTION}

Quadric surfaces of revolution - ellipsoids, cones, paraboloids, cylinders, and hyperboloids - are the most basic non-trivial surfaces in $\mathbf{R}^{3}$. Euler noted [B] that one can describe them all, up to rigid motion, by appropriately choosing constants $a, b, c$ in the simple quadratic equation

$$
x^{2}+y^{2}=a z^{2}+b z+c .
$$

The left side of this equation measures distance (squared) from the $z$ axis - the axis of revolution - while the right sets the vertical "profile" of the surface.

Archimedes knew that any plane tilted sufficiently far from the axis of such a surface cuts it in an ellipse, and Fermat apparently realized the converse: That elliptical cross-sections make a surface of revolution quadric [C]. One can verify Fermat's observation using high-school algebra, but here we prove a far stronger statement: Cross-sections which are merely central (in the sense we shortly explain) make a surface of revolution quadric.

This fact has generalizations and consequences, some of which we will sketch in our concluding remarks, but we leave the full treatment of

Date: First draft October 2007. Last Typeset March 1, 2019.

Key words and phrases. Quadric surface, surface of revolution, central symmetry, convex loop.

Supported by a Lady Davis fellowship. 
such extensions to a forthcoming article. Here we focus entirely on the claim above. We proceed now to define the terms we need to state and prove it with precision.

By central, we mean that a cross-section has symmetry with respect to reflection through its center of mass - its centroid. Circles, ellipses, and many other loops have central symmetry, though of course most loops do not. For instance, any loop sufficiently close to a triangle lacks it.

A $C^{1}$ loop in $\mathbf{R}^{3}$ is the image of a differentiably immersed circle.

By a $C^{1}$ surface of revolution, we mean a surface $S$ definable, after a rigid motion, as the locus

$$
x^{2}+y^{2}=F(z), \quad|z|<q .
$$

Here $F$ is a strictly positive, differentiable "profile" function, and $0<$ $q \leq \infty$ bounds the vertical extent of the surface. To indicate that a surface of revolution has this description for some $q>0$ and some $F:(-q, q) \rightarrow(0, \infty)$, we say it lies in standard position. Note that we allow $F(z) \rightarrow \infty$ as $|z| \rightarrow q$. This can cause technical complications that we will avoid, when necessary, by focusing on the restricted surface

$$
S_{\delta}:=\{(x, y, z) \in S:|z|<q-\delta\} .
$$

We deem a plane $P$ transverse to $S$ if $P \cap S \neq \emptyset$, and $P$ never coincides with the tangent plane of $S$ at any point of their intersection.

All planes of interest here take the "graphical" form

$$
z=m_{1} x+m_{2} y+\beta \text {. }
$$

We call $m:=\sqrt{m_{1}^{2}+m_{2}^{2}}$ the slope, and $\beta$ the intercept of this plane, which we henceforth denote by $P_{m, \beta}$. Actually, an entire circle of planes have slope $m$ and intercept $\beta$, but the rotational symmetry of our problem makes them all equivalent for our purposes; we can safely ignore the ambiguity.

Theorem 1.1 (Main result). Suppose we have a $C^{1}$ surface of revolution $S$ in standard position, and for some $\mu>0$, every plane of slope $m<\mu$ that cuts $S$ in a loop does so in central loop. Then $S$ is quadric. 


\section{The ARgument}

We prepare to prove our main result with three simple lemmas. The first merely records some basic facts about surfaces of revolution:

Lemma 2.1. Suppose a $C^{1}$ surface of revolution $S$ lies in standard position, and $\delta>0$. Then there exists $\mu>0$ such that $P_{m, \beta} \cap S_{\delta}$ is a $C^{1}$ loop lying in the slab $|z-\beta|<\delta$ whenever $m<\mu$ and $|\beta|<q-2 \delta$.

One proves this using the implicit function theorem, together with the boundedness of $F$ and $F^{\prime}$ when $|z|<q-\delta$. The result seems geometrically evident in any case, so we leave further details to the reader.

The next lemma puts an amusing gloss on the classical Mean Value Theorem for a differentiable function $f$. The latter equates the slope of the chord between $(a, f(a))$ and $(b, f(b))$ to the derivative $f^{\prime}(c)$ for some $c$ between $a$ and $b$. If we insist that $c$ coincide always with the midpoint of $a$ and $b$, it turns out that we make $f$ quadratic:

Lemma 2.2. Suppose $f$ is differentiable on an open interval $I$, and for some $\varepsilon>0$ and all $\zeta \in I$, we have

$$
f^{\prime}(\zeta)=\frac{f(\zeta+t)-f(\zeta-t)}{2 t}
$$

whenever $|t|<\varepsilon$ and $\zeta \pm t \in I$. Then $f$ is quadratic on $I$.

Proof. Our assumptions make the right-hand side of (2.1) - and hence the left too - differentiable with respect to $\zeta$ when $|t|<\varepsilon$ and $\zeta \pm$ $t \in I$. For such $t$, taking $d / d \zeta$ has the effect of replacing $f$ by $f^{\prime}$ throughout (2.1). It follows that $f^{\prime \prime}$, and by iteration, all derivatives of $f$, exist as continuous functions on $I$.

Now multiply (2.1) by $2 t$ and, under the same harmless restrictions on $t$, differentiate thrice, this time with respect to $t$. One gets

$$
0=f^{\prime \prime \prime}(\zeta+t)+f^{\prime \prime \prime}(\zeta-t) .
$$

Setting $t=0$ now shows that $f^{\prime \prime \prime} \equiv 0$ on $I$.

The conclusion of the next lemma should now look promising. Recall that $q>0$ measures the vertical extent of a surface of revolution $S$ in standard position. 
Lemma 2.3. Suppose $S$ is a surface of revolution in standard position, $m>0$, and that for some $\beta \in(-q, q)$ the intersection $P_{m, \beta} \cap S$ is a central loop centered at height $\zeta$. Then

$$
F^{\prime}(\zeta)=\frac{F(\zeta+t)-F(\zeta-t)}{2 t}
$$

whenever $|t|<\sup \left\{z-\zeta:(x, y, z) \in P_{m, \beta} \cap S\right\}$.

Proof. Define $b:=-\beta$. The rotational symmetry of $S$ then lets us assume our plane $P_{m, \beta}$ takes the form

$$
z=m x-b
$$

and by using this equation to eliminate $x$ in (1.2), we can characterize our loop $P_{m, \beta} \cap S$, in the $(y, z)$-coordinate system on $P_{m, \beta}$, as the locus

$$
\left(\frac{b+z}{m}\right)^{2}+y^{2}=F(z) .
$$

Clearly, the reflection

$$
(y, z) \longmapsto(-y, z)
$$

preserves this loop, and in particular, $y=0$ at its centroid. We can therefore express its assumed central symmetry by

$$
(y, z) \longmapsto(-y, 2 \zeta-z) \text {. }
$$

The key point is that by composing the bilateral and central reflections above, we obtain a further bilateral symmetry, namely

$$
(y, z) \longmapsto(y, 2 \zeta-z) \text {. }
$$

But the $y \leftrightarrow-y$ symmetry (2.4) must clearly split our loop $P_{m, \beta} \cap S$ into two congruent arcs, one of which lies in the half-plane $y \geq 0$. If we translate the origin to the centroid of our loop via the coordinatechange $z=\zeta+t$, then by (2.3), we can describe that arc in $(t, y)$ coordinates as the graph of

$$
y=\sqrt{F(\zeta+t)-\left(\frac{b+\zeta+t}{m}\right)^{2}} .
$$

Since the additional symmetry (2.5) maps $(y, \zeta+t)$ to $(y, \zeta-t)$, however, we can write this same arc as the graph of

$$
y=\sqrt{F(\zeta-t)-\left(\frac{b+\zeta-t}{m}\right)^{2}} .
$$


Squaring and equating the two expressions for $y$ in (2.6) and (2.7), we now get

$$
F(\zeta+t)-\left(\frac{b+\zeta+t}{m}\right)^{2}=F(\zeta-t)-\left(\frac{b+\zeta-t}{m}\right)^{2}
$$

valid whenever $|t|<\sup \left\{z-\zeta:(x, y, z) \in P_{m, \beta} \cap S\right\}$. The expression above then simplifies to

$$
\frac{2(b+\zeta)}{m^{2}}=\frac{F(\zeta+t)-F(\zeta-t)}{2 t}
$$

and by letting $t \rightarrow 0$, we see that the constant on the left must equal $F^{\prime}(\zeta)$. This proves the lemma.

We can now verify our main result.

Proof of Theorem 1.1. We must show that $F$ is quadratic. To do so, let $\delta>0$. Then our assumptions, together with that of Lemma 2.1, guarantee that for some small but positive slope $m>0$, the plane $P_{m, \beta}$ given by

$$
z=m x+\beta
$$

cuts $S_{\delta}$ in a central loop lying in the slab $|z-\beta|<\delta$, provided only that $|\beta|<q-2 \delta$.

In particular, if we define a function

$$
\zeta:(-q+2 \delta, q-2 \delta) \rightarrow \mathbf{R}
$$

by making $\zeta(\beta)$ equal the height of the centroid of the loop $P_{m, \beta} \cap S_{\delta}$, the image of this function must contain the entire interval $|z|<q-3 \delta$.

Note also that the continuity and positivity of $F$ for $|z|<q$ ensure that

$$
\phi(\delta):=\inf \{\sqrt{F(z)}:|z|<q-\delta\}>0 .
$$

This means in particular that when $|\beta|<q-2 \delta$, the loop $P_{m, \beta} \cap S_{\delta}$ lies outside the cylinder $x^{2}+y^{2}=\phi(\delta)$, so that the extreme values of $z$ on this loop differ by at least $2 m \phi(\delta)$. These extrema then deviate from $\zeta(\beta)$ - the height of the loop centroid - by at least

$$
\varepsilon:=m \phi(\delta) .
$$

Lemma 2.3 now ensures that on the interval $|\beta|<q-3 \delta$, our profile function $F$ satisfies the assumptions of Lemma 2.2 with the value of $\varepsilon$ just determined, making $F$ quadratic on this interval. But $\delta$ was 
arbitrary, so $F$ is quadratic for all $|z|<q$, and hence $S$ is quadric, as claimed.

Remark 2.4. Though stated in $\mathbf{R}^{3}$, all arguments above, and indeed our Main Theorem, generalize immediately to higher dimensions. One simply introduces coordinates

$$
\left(x_{1}, \cdots, x_{n-2}, y, z\right) \in \mathbf{R}^{n},
$$

and replaces $x^{2}$ by $|x|^{2}:=\sum_{i=1}^{n-2} x_{i}^{2}$ in (1.2) to define hypersurface of revolution in standard position. Everything above then generalizes to $\mathbf{R}^{n}$, with the word "surface" replaced by "hypersurface" throughout.

Remark 2.5. In a forthcoming paper, we apply the main result here in an essential way to prove a far more general result. Roughly speaking, we show there that any surface in $\mathbf{R}^{3}$ which has compact, convex planar cross-sections, all of them central, must be quadric.

Remark 2.6. A skewloop is $C^{1}$ loop in $\mathbf{R}^{3}$ without any pair of parallel tangent lines. The term was coined in [GS], which goes on to show that convex quadrics are the only positively curved surfaces without skewloops. Because our Main Theorem says that every nonquadric surface of revolution has an non-central cross-section, one can exploit the "grafting" technique from [GS, §5] to show:

\section{Every non-quadric surface of revolution in $\mathbf{R}^{3}$ admits a} skewloop.

Conversely, by [SS] (or, generically, [T]), no quadric admits a skewloop. So the Main Theorem here yields a characterization of the one-sheeted hyperboloid (i.e. the case where $a>0$ in (1.1)) as the only negatively curved surface of revolution without skewloops. The generalization described in Remark 2.5 will remove the need to assume rotational symmetry. Still, the Main Theorem here yields a first negatively curved counterpart to the characterization of positively curved quadrics in GS.

\section{ACKNOWLEDGMENTS}

We thank the Lady Davis Foundation, the Technion (Israel Institute of Technology), and Indiana University for supporting this work. 
CENTRAL CROSS-SECTIONS MAKE SURFACES OF REVOLUTION QUADRIC7

\section{REFERENCES}

[Bo] C.B. Boyer, History of Analytic Geometry, Scripta Mathematica, New York, 1956.

[C] J.L. Coolidge, The Origin of Analytic Geometry, Osiris 1 1936, 231-250. (Also available at www.jstor.org)

[GS] M. Ghomi \& B. Solomon, Skew loops and quadric surfaces, Comment. Math. Helv. 77 (2002), 4, 767-782.

[SS] J.-P. Sha \& B. Solomon, No skew branes on non-degenerate hyperquadrics, Math. Zeit. 257:225-229 (2007)

[T] S. Tabachnikov, On skew loops, skew branes, and quadratic hypersurfaces, Moscow Math. J. 3, 681-690 (2003).

Math Department, Indiana University, Bloomington IN 47405

E-mail address: solomon@indiana.edu

$U R L$ : mypage.iu.edu/ solomon 